\title{
Quantisation of second class systems in the Batalin-Tyutin formalism
}

\author{
N. Banerjee" \\ Saha Institute of Nuclear Physics, 1/AF Bidhannagar \\ Calcutta 700064, India \\ R. Banerjee \\ S. N. Bose National center for Basic Sciences \\ DB-17, Salt Lake, Calcutta 700064, India \\ Subir Ghosh \\ Gobardanga Hindu College, North 24 Parganas \\ West Bengal, India
}

\begin{abstract}
We review the Batalin-Tyutin approach of quantising second class systems which consists in enlarging the phase space to convert such systems into first class. The quantisation of first class systems, it may be mentioned, is already well founded. We show how the usual analysis of Batalin-Tyutin may be generalised, particularly if one is dealing with nonabelian theories. In order to gain a deeper insight into the formalism we have considered two specific examples of second class theories- the massive Maxwell theory (Proca model) and its nonabelian extension. The first class constraints and the involutive Hamiltonian are explicitly constructed. The connection of our Hamiltonian approach with the usual Lagrangian formalism is elucidated. For the Proca model we reveal the importance of a boundary term which plays a significant role in establishing an exact identification of the extra fields in the Batalin-Tyutin approach with the Stückelberg scalar. Some comments are also made concerning the corresponding identification in the nonabelian example.
\end{abstract}

\footnotetext{
${ }^{1}$ e-mail address: shila@saha.ernet.in
} 


\section{Introduction}

Canonical quantisation of systems with first class constraints was formulated along general lines by Dirac [1]. The corresponding analysis in the path integral approach was initiated by Faddeev [2] for gauge theories. It was extended by Fradkin and collaborators [3, 4] within the broader framework of preserving Becchi-Rouet-Stora-Tyutin (BRST) [5] invariance. The quantisation of systems with second class constraints, on the contrary, poses problems. In this case it is necessary to replace the canonical Poisson brackets (P.B) by their corresponding Dirac brackets (D.B). The conversion of the D.B's to quantum commutators is, in general, plagued with severe factor ordering problems. Moreover, the abstraction of the canonically conjugate variables is highly nontrivial. Consequently the quantisation of second class systems, either in the canonical or in the path integral formalisms, is problematic. It may be mentioned that ther is a factor ordering problem of a different nature in first class systems. There are three varities of this problem. First, the constraints which close under P.B may do so with coefficients which are functions of the canonical variables rather than just structure constants. Second, the P.B of the constraints with the Hamiltonian may yield a combination of constraints with phase space coefficients. Finally, the Poisson algebra of the constraints with any physical variable must yield a combination of constraints which may involve structure functions instead of structure constants. In all these cases a suitable factor ordering has to be found such that the structure function operators precede the correctly ordered constraints [1, 6]. It is only then possibly to develop a consistent quantisation program. This has been done by Kuchar [6] in a covariant manner. The factor ordering concerning the interpretation of the D.B as a quantum commutator remains an open issue.

In view of the above discussion it becomes natural to formulate the quantisation of second class systems without invoking D.B. A possible way would be to embed such a system in an extended space so that it gets converted into first class. One can then apply the well established machinery [3, 4, 5] for quantising first class systems. This philosophy has been recently adopted by Batalin-Fradkin [7] and Batalin-Tyutin [8]. The phase space is extended by introducing new variables which transform the original second class system into first class. It is worthwhile to mention that this idea is a logical follow up of the original notion of Stückelberg [9] who extended the configu- 
ration space to convert second class theories into first class. The approach of Stückelberg [9] is in the Lagrangian formulation which should be contrasted with the Hamiltonian formulation of Batalin-Fradkin [7] or Batalin-Tyutin [8]. A similar (Lagrangian) functional integral approach has been used by Faddeev and Satashvili [10] to introduce Wess-Zumino scalars [11] to interpret anomalous gauge theories as true (i.e. first class) gauge systems. Recently there have been suggestions [12, 13] that the extra fields introduced in the Hamiltonian formalism may be identified with the Stückelberg scalar or the Wess-Zumino fields. We will return to this point later.

The purpose of this paper is to make a thorough investigation into the Hamiltonian formulation of Batalin-Tyutin [8] to convert second class systems into first class, by referring to two examples- the abelian (Proca) model and its nonabelian extension. In the course of this analysis the precise connection with the Lagrangian formulation as well as the identification of the extra fields with the Stückelberg [9] scalar will also be elucidated.

In section 2 we first briefly review the analysis of Batalin-Tyutin [ 8$]$ ]. This will serve a twofold purpose; to set up the notation and familiarise the reader with the basics of the formalism. This formalism [8] is ideal for discussing abelian (second class) systems. To appreciate this point it may be recalled that, for abelian first class systems, the usual algebra of constraints among themselves and with the Hamiltonian is strongly involutive. The method developed in [8] also yields a strongly involutive algebra for first class systems. For nonabelian (first class) theories, on the other hand, it is well known that the corresponding algebra is only weakly involutive. We, therefore, generalise the approach of [8] to include this possibility. This has been discussed in great details. Its utility is made transparent when we actually convert a nonabelian second class theory into first class. We are able to reproduce the algebra which occurs in usual nonabelian first class theories. If the standard proceedure of [8] were adopted we would obviously fail to generate this algebra. This will also have some implications in connecting the Hamiltonian formalism with the Lagrangian version.

Section 3 is devoted to an application of the ideas discussed in section 2 to the abelian Proca model [9]. The original second class system is transformed into first class by initially converting the second class constraints to first class and then changing the Hamiltonian into the corresponding involutive form. The phase space partition function is constructed and explicitly evaluated in two special gauges. In the unitary gauge [7, 8] which corresponds to 
taking the initial second class constraints as the gauge fixing conditions, the original theory is reproduced. Nontrivial consequences are obtained in the Faddeev-Popov [14] like gauges (i.e. gauges not involving the momenta). It leads to a Lagrangian embedding which reveals the first class nature of the theory. The connection with the conventional Stückelberg 99 Lagrangian mechanism is established. It is shown how a boundary term plays a crucial role in making a one-to-one correspondence between the extra field in the Hamiltonian formalism with the Stückelberg [9] scalar.

In section 4 we introduce the nonabelian version of the Proca model. We first point out a flaw in the conventional Dirac analysis performed by Senjanovic [15. The correct D.B's among the canonical variables are worked out. These are found to be field dependent. We next exploitthe generalised version of the Batalin-Tyutin [8] approach developed in section 2 to convert thye nonabelian second class system into first class. It is interesting to observe that while one of the (first class) constraints has a closed form, the other constraint and the involutive Hamiltonian are expressed by a power series in terms of the new fields and do not have any closed expressions. Specialising to the case where the gauge group is $S U(2)$, we are able to reproduce the gauge algebra given in the conventional Lagrangian formulation [16] obtained by the nonabelian Stückelberg mechanism. Although the identification of the extra fields in the Hamiltonian formulation with the Stc̈kelberg fields (which now appear as the Euler angles [16]) in the Lagrangian approach is not so direct as in the abelian example, nevertheless there is no conceptual difficulty in understanding this correspondence. Finally we show that contrary to claims in the literature [17], the nonabelian extension of the usual Stückelberg [9] mechanism fails to convert the nonabelian second class theory into first class.

Our concluding observations are given in section 5 .

\section{General formalism}

In this section we first review the abelian conversion of a second class system into first class as developed by Batalin and Tyutin (BT) [8]. We then discuss a natural extension of this approach which is suitable for analysing

nonabelian models. Simultaneously our notations and conventions will also 
be specified.

Let us assume that the canonical variables, $\left(\phi_{i}(x), \pi^{i}(x)\right)$ with $|i|=n$ and a Grassman parity $\epsilon\left(\phi_{i}\right)=\epsilon\left(\pi^{i}\right)=\epsilon_{i}$ define the initial phase space of a dynamical system. We further suppose that this system contains a set of linearly independent bosonic second class constraints $\Theta_{\alpha}$ which are some functions of the original canonical variables.

$$
\Theta_{\alpha}=\Theta_{\alpha}(\phi, \pi), \epsilon_{\alpha}=0,|\alpha|=m<2 n
$$

so that the matrix,

$$
\Delta_{\alpha \beta}(x, y)=\left\{\Theta_{\alpha}(x), \Theta_{\beta}(y)\right\}
$$

has a nonvanishing determinant. The inclusion of other constraints (i.e. fermion or first class) is a matter of technical detail and poses no problems in developing the formalism.

We now convert the second class system into first class. The initial step is to obtain the first class constraints starting from (11). Foliowing the general philosophy, new dynamical fields,

$$
\Phi^{\alpha}, \quad \epsilon_{\alpha}=0, \quad|\alpha|=m
$$

with the basic P.Bs,

$$
\left\{\Phi^{\alpha}(x), \quad \Phi^{\beta}(y)\right\}=\omega^{\alpha \beta}(x, y)
$$

where $\omega$ is an invertible field independent antisymmetric matrix,

$$
\omega^{\alpha \beta}(x, y)=-\omega^{\beta \alpha}(y, x), \quad \epsilon\left(\omega^{\alpha \beta}\right)=\epsilon_{\alpha}+\epsilon_{\beta},
$$

are introduced to extend the original phase space,

$$
(\phi, \pi) \oplus(\Phi) \text {. }
$$

The new first class constraints of the system in the extended phase space (6) are denoted by $\tau_{\alpha}$ :

$$
\tau_{\alpha}=\tau_{\alpha}(\phi, \pi, \Phi) ; \quad \epsilon\left(\tau_{\alpha}\right)=\epsilon_{\alpha},|\alpha|=m
$$

Then the abelian conversion of BT [8] implies that these constraints are strongly involutive,

$$
\left\{\tau_{\alpha}(x), \tau_{\beta}(y)\right\}=0
$$


subject to the boundary condition,

$$
\tau_{\alpha}(\phi, \pi, 0)=\Theta_{\alpha}(\phi, \pi)
$$

where the right hand side of (9) is just the original set of second class constraints (11).

In ref. [8] it has been shown that it is possible to explicitly construct the solution to the algebraic problem (8) and (9) in the form of a power series expansion,

$$
\tau_{\alpha}(\phi, \pi, \Phi)=\sum_{n=0}^{\infty} \tau_{\alpha}^{(n)}, \quad \tau_{\alpha}^{(n)} \sim \Phi^{n}
$$

The first term yields (9)

$$
\tau_{\alpha}^{(0)}=\Theta_{\alpha}
$$

The remaining terms are found by inserting (10) in (8), using the relation (2) and finally identifying contributions with identical powers of $\Phi^{\alpha}$. This leads to the following recursion relations,

$$
\begin{aligned}
\left\{\tau_{\alpha}^{(0)}(x), \tau_{\beta}^{(0)}(y)\right\}_{(\phi, \pi)}+\left\{\tau_{\alpha}^{(1)}(x), \tau_{\beta}^{(1)}(y)\right\}_{(\Phi)} & =0 \\
\left\{\tau_{[\alpha}^{(1)}(x), \tau_{\beta]}^{(n+1)}(y)\right\}_{(\Phi)}+B_{\alpha \beta}^{(n)}(x, y) & =0, \quad n \geq 1
\end{aligned}
$$

with,

$$
\begin{aligned}
B_{\alpha \beta}^{(1)}(x, y) & \equiv\left\{\tau_{[\alpha}^{(0)}(x), \tau_{\beta]}^{(1)}(y)\right\}_{(\phi, \pi)} \\
B_{\alpha \beta}^{(n)}(x, y) \equiv \frac{1}{2} B_{[\alpha \beta]}^{(n)}(x, y) & \equiv \sum_{m=0}^{n}\left\{\tau_{\alpha}^{(n-m)}(x), \tau_{\beta}^{(m)}(y)\right\}_{(\phi, \pi)} \\
& +\sum_{m=0}^{n-2}\left\{\tau_{\alpha}^{(n-m)}(x), \tau_{\beta}^{(m+2)}(y)\right\}_{(\Phi)}, \quad(n \geq 2)(15
\end{aligned}
$$

where the suffix $(\phi, \pi)$ or $(\Phi)$ denotes the variables with respect to which the corresponding P.B. are evaluated. It is now straightforward to explicitly construct the individual terms in the series (10). For example, expanding $\tau_{\alpha}^{(1)}$ as,

$$
\tau_{\alpha}^{(1)}(x)=\int d y X_{\alpha \beta}(x, y) \Phi^{\beta}(y)
$$

and exploiting (2), (11) and (13) it is found that $X_{\alpha \beta}(x, y)$ is given by,

$$
\int X_{\alpha \mu}(x, z) \omega^{\mu \nu}\left(z, z^{\prime}\right) X_{\beta \nu}\left(y, z^{\prime}\right) d z d z^{\prime}=-\Delta_{\alpha \beta}(x, y)
$$


where $\Delta_{\alpha \beta}$ is defined in (2). This determines $\tau_{\alpha}^{(1)}$ (16).

In order to obtained the complete series (10) it is essential to introduce the matrix $\omega_{\alpha \beta}$ and $X^{\alpha \beta}$ which are inverse to $\omega^{\alpha \beta}$ and $X_{\alpha \beta}$ respectively,

$$
\begin{aligned}
\int \omega^{\alpha \beta}(x, y) \omega_{\beta \gamma}(y, z) d y & =\delta_{\gamma}^{\alpha} \delta(x-z) \\
\int X^{\alpha \beta}(x, y) X_{\beta \gamma}(y, z) d y & =\delta_{\gamma}^{\alpha} \delta(x-z)
\end{aligned}
$$

Then the particular solution of the inhomogeneous equation (13) is given by,

$\tau_{\alpha}^{(n+1)}(x)=-\frac{1}{n+2} \int \Phi^{\mu}(z) \omega_{\mu \nu}\left(z, z^{\prime}\right) X^{\nu \rho}\left(z^{\prime}, z^{\prime \prime}\right) B_{\rho \alpha}^{(n)}\left(z^{\prime \prime}, x\right) d z d z^{\prime} d z^{\prime \prime} \quad(n \geq 1)$

The general solution to (13) can be obtained from (19) by adding to it a term containing the solution of the homogeneous equation corresponding to (13). It has been shown [8] that any arbitrariness in these solutions corresponds to canonical transformations in the extended phase space. For actual computational purpose, therefore, it suffices to work with the solution (16) and (19).

It may be remarked that this arbitrariness in constructing the solution of the first class constraints is reminiscent of an analogous feature in the conventional 'splitting' approach of regarding a second class system as a gauged fixed first class one. The latter, it may be recalled, consists in interpreting one half of the second class constraints (11) as first class ones and the other half as the corresponding gauge fixing conditions. Clearly there is an arbitrariness in this splitting which is also manifested in the ensuing symplectic structure.

The construction of the strongly involutive set of constraints (10) completes the first part of the program. It is now necessary to construct the corresponding involutive Hamiltonian $\tilde{H}$ which will be defined in the extended phase space,

$$
\tilde{H}=\tilde{H}(\phi, \pi, \Phi)
$$

In the spirit of BT's approach, strong involution (or 'abelianisation) is required,

$$
\left\{\tau_{\alpha}(x), \tilde{H}\right\}=0
$$

subject to the boundary condition,

$$
\tilde{H}(\phi, \pi, 0)=H_{0}(\phi, \pi)
$$


where $H_{0}(\phi, \pi)$ is the original Hamiltonian, and $\tau_{\alpha}$ in (21) are the involutive constraints. There is a degree of freedom in choosing the original Hamiltonian $H_{0}$. This may just be the usual canonical Hamiltonian. Alternatively, it may be the total Hamiltonian obtained from the canonical part by adding terms proportional to the second class constraints with the Lagrange multipliers (i.e. the proportionality constants) determined by the usual manner of Dirac [1]. The particular choice of $H_{0}$ is crucial for technical reasons resulting in considerable algebraic simplifications, and depends on the specific model being studied.

Expressing the solution to (21) as a power series,

$$
\tilde{H}=\sum_{n=0}^{\infty} \tilde{H}^{(n)}, \quad \tilde{H}^{(n)} \sim \Phi^{n}
$$

with,

$$
\tilde{H}^{(0)}(\phi, \pi ; \Phi)=\tilde{H}(\phi, \pi ; 0)=H_{0}(\phi, \pi)
$$

Substituting (23) and (10) in (21), the following set of recursion relations are obtained [8],

$$
\left\{\tau_{\alpha}^{(1)}(x), \tilde{H}^{(n+1)}\right\}_{(\Phi)}+G_{\alpha}^{(n)}(x)=0, \quad n \geq 0
$$

where,

$$
\begin{aligned}
& G_{\alpha}^{(0)} \equiv\left\{\tau_{\alpha}^{(0)}, \tilde{H}^{(0)}\right\} \\
& G_{\alpha}^{(1)} \equiv\left\{\tau_{\alpha}^{(1)}, \tilde{H}^{(0)}\right\}+\left\{\tau_{\alpha}^{(0)}, \tilde{H}^{(1)}\right\}+\left\{\tau_{\alpha}^{(2)}, \tilde{H}^{(1)}\right\}_{(\Phi)} \\
& G_{\alpha}^{(n)} \equiv \sum_{m=0}^{n}\left\{\tau_{\alpha}^{(n-m)}, \tilde{H}^{(m)}\right\}+\sum_{m=0}^{n-2}\left\{\tau_{\alpha}^{(n-m)}, \tilde{H}^{(m+2)}\right\}_{(\Phi)}+\left\{\tau_{\alpha}^{(n+1)}, \tilde{H}^{(1)}\right\}_{(\Phi)}, \quad n \geq 2
\end{aligned}
$$

It is now possible to give the solution to the inhomogeneous equation (25),

$$
\tilde{H}^{(n+1)}=-\frac{1}{n+1} \int \Phi^{\mu}(x) \omega_{\mu \nu}(x, y) X^{\nu \rho}(y, z) G_{\rho}^{(n)}(z) d x d y d z, \quad(n \geq 0)
$$

which gives the involutive Hamiltonian. This completes the BT [8] construction of the first class system which is strongly involutive.

We now analyse the characteristics of this construction and the need for some modifications. Note that the strongly involutive algebra characterises a rank zero theory [4]. This is rather restrictive since, in certain cases, a more natural representation could be done in terms of higher rank theories. 
Although the rank of a theory may not be unique, yet there may be some conceptual or technical problems in - say - expressing a rank one theory as a rank zero type. Let us illuminate by an example. It is usual to regard the Yang-Mills theory as of rank one since the algebra of the first class (Gauss) constraint is only weakly involutive, expressing the standard closure property. Now it may be possible to write down a linear combination of the first class constraints (which would also be first class constraint) so that these are strongly involutive thereby rendering the Yang-Mills theory as rank zero. In that case, however, apart from arcane algebraic structures the natural identity of the first class constraint with the zero component of the equation of motion gets lost and its simple interpretation as the generator of the gauge transformation may also be difficult to preserve. Keeping these points in view, therefore, it is desirable to extend the BT formalism to represent higher rank theories by modifying the involutive algebra (8) as ,

$$
\left\{\tau_{\alpha}^{a}(x), \tau_{\beta}^{b}(y)\right\}=f^{a b c} \int \Gamma_{\alpha \beta \gamma}(x, y, z) \tau_{\gamma}^{c}(z) d z
$$

where the structure functions are field independent. This characterises a rank one theory. A rank two theory would occur if the structure functions are field dependent. This happens in the case of gravity which is kept outside the realm of the present paper. The algebra (30) is ideal for discussing nonabelian theories where $a, b, c$ represent the corresponding group indices. Note that, in the abelian case, (30) goes over to (8).

Following similar techniques as BT [8] by expressing $\tau_{\alpha}^{a}(x)$ as a power series analogous to (10),

$$
\tau_{\alpha}^{a}=\sum_{n=0}^{\infty} \tau_{\alpha}^{a(n)} ; \quad \tau_{\alpha}^{a(n)} \sim\left(\Phi^{\alpha a}\right)^{n}
$$

we obtain the following relations corresponding to (13)-(15) with the appropriate group indices,

$$
\begin{aligned}
&\left\{\tau_{\alpha}^{a(0)}(x), \tau_{\beta}^{b(0)}(y)\right\}+\left\{\tau_{\alpha}^{a(1)}(x), \tau_{\beta}^{b(1)}(y)\right\}_{(\Phi)} \\
&= f^{a b c} \int \Gamma_{\alpha \beta \gamma}(x, y, z) \tau_{\gamma}^{c(0)}(z) d z \\
&\left\{\tau_{[\alpha}^{a(1)}(x), \tau_{\beta]}^{b(n+1)}(y)\right\}_{(\Phi)}+B_{\alpha \beta}^{a b(n)}(x, y)=0, \quad n \geq 1
\end{aligned}
$$


where,

$$
\begin{aligned}
B_{\alpha \beta}^{a b(1)}(x, y) & =\left\{\tau_{[\alpha}^{a(0)}(x), \tau_{\beta]}^{b(1)}(y)\right\}-f^{a b c} \int \Gamma_{\alpha \beta \gamma}(x, y, z) \tau_{\gamma}^{c(1)}(z) d z \\
B_{\alpha \beta}^{a b(n)}(x, y) & =\sum_{m=0}^{n}\left\{\tau_{\alpha}^{a(n-m)}(x), \tau_{\beta}^{b(m)}(y)\right\}+\sum_{m=0}^{n-2}\left\{\tau_{\alpha}^{a(n-m)}(x), \tau_{\beta}^{b(m+2)}(y)\right\}_{(\Phi)} \\
& -f^{a b c} \int \Gamma_{\alpha \beta \gamma}(x, y, z) \tau_{\gamma}^{c(n)}(z) d z, \quad n \geq 2
\end{aligned}
$$

It may be checked that the required solution of the inhomogeneous equation (33) is given by,

$$
\begin{gathered}
\tau_{\alpha}^{a(0)}=\Theta_{\alpha}^{a} \\
\tau_{\alpha}^{a(1)}(x)=\int d y X_{\alpha \beta}^{a b}(x, y) \Phi^{b \beta}(y)
\end{gathered}
$$

with,

$$
\begin{aligned}
\int X_{\alpha \mu}^{a c}(x, z) \omega_{c d}^{\mu \nu}\left(z, z^{\prime}\right)- & X_{\beta \nu}^{b d}\left(y, z^{\prime}\right) d z d z^{\prime} \\
- & f^{a b c} \int \Gamma_{\alpha \beta \gamma}(x, y, z) \Theta_{\gamma}^{c}(z)=-\Delta_{\alpha \beta}^{a b}(x, y)
\end{aligned}
$$

and,

$$
\tau_{\alpha}^{a(n+1)}(x)=-\frac{1}{n+2} \int \Phi^{\mu b}(z) \omega_{\mu \nu}^{b c}\left(z, z^{\prime}\right) X_{c d}^{\nu \rho}\left(z^{\prime}, \quad z^{\prime \prime}\right) B_{\rho \alpha}^{d a(n)}\left(z^{\prime \prime}, x\right) d z d z^{\prime} d z^{\prime \prime}
$$

where $\Delta_{\alpha \beta}^{a b}, \omega_{\mu \nu}^{a b}, X_{a b}^{\mu \nu}$ are the analogues of (2), (18) with the appropriate group indices. This completes the constructions of the involutive constraints (31) satisfying the algebra (30). A similar analysis can be done for the Hamiltonian which is straightforward and not repeted.

\section{An abelian example- the Proca model}

It is instructive to apply the general ideas discussed in the previous section to specific models. Such model based analysis clarifies several issues and gives 
a deeper insight into the general formalism. Let us first consider a simple abelian example which is the Proca model whose dynamics is governed by the Lagrangian density,

$$
\mathcal{L}=-\frac{1}{4} F_{\mu \nu} F^{\mu \nu}+\frac{1}{2} m^{2} A^{\mu} A_{\mu}
$$

Note the introduction of an explicit mass term which breaks the gauge invariance of the usual Maewell's theory. Consequently (40) represents a second class system which is easily confirmed by the standard constraint analysis. There is one primary constraint,

$$
\Theta_{1}=\pi_{0} \approx 0
$$

and one secondary constraint,

$$
\Theta_{2}=\partial_{i} \pi^{i}+m^{2} A_{0} \approx 0
$$

obtained by conserving $\Theta_{1}$ with the total Hamiltonian,

$$
H_{T}=H_{c}+\int d^{3} x \lambda \Theta_{1}
$$

where $H_{c}$ is the canonical Hamiltonian,

$$
H_{c}=\int\left[\frac{1}{2} \pi_{i}^{2}+\frac{1}{4} F_{i j}^{2}+\frac{1}{2}\left(A_{0}^{2}+A_{i}^{2}\right)-A_{0} \Theta_{2}\right]
$$

and $\lambda$ is a Lagrange multiplier, while $\pi_{\mu}$ is the momenta canonically conjugate to $A^{\mu}$. The algebra,

$$
\Delta_{\alpha \beta}(x, y)=\left\{\Theta_{\alpha}, \Theta_{\beta}\right\}=m^{2} \epsilon_{\alpha \beta} \delta(x-y) ; \quad \alpha, \beta=1,2
$$

where the antisymmetric tensor $\epsilon_{\alpha \beta}$ is normalised as,

$$
\epsilon_{12}=-\epsilon^{12}=-1
$$

reveals the second class nature of the constraints $\Theta_{\alpha}(x)$.

In order to convert this system into first class, the first objective is to transform $\Theta_{\alpha}$ into first class by extending the phase space. Following the general approach we have to introduce the matrix (5), which is chosen as,

$$
\omega^{\alpha \beta}(x, y)=2 \epsilon^{\alpha \beta} \delta(x-y)
$$


Then the other matrix $X_{\alpha \beta}$ in the game is obtained by solving (17) with $\Delta_{\alpha \beta}$ given by (45),

$$
X_{\alpha \beta}(x, y)=\left(\begin{array}{rr}
1 & 0 \\
0 & \frac{m^{2}}{2}
\end{array}\right) \delta(x-y)
$$

There is an arbitrariness in choosing $\omega^{\alpha \beta}$ (47) which would naturally be manifested in (48). This just corresponds to canonical transformations in the extended phase space. However, as has also been evidenced in other calculations [19, 20, 21], the choice (47) brings about remarkable algebraic simplifications.

Using (10), (16) and (48) the new set of constraints are found to be,

$$
\begin{aligned}
\tau_{1} & =\Theta_{1}+\Phi^{1} \\
\tau_{2} & =\Theta_{2}+\frac{m^{2}}{2} \Phi^{2}
\end{aligned}
$$

which are strongly involutive,

$$
\left\{\tau_{\alpha}, \tau_{\beta}\right\}=0
$$

Recall the $\Phi^{1}, \Phi^{2}$ are the new variables satisfying the algebra (雨) with $\omega^{\alpha \beta}$ given by (47). Observe further that only $\tau_{\alpha}^{(1)}$ (16) contributes in the series (10) defining the first class constraint. All higher order terms given by (19) vanish as a consequence of our intelligent choice (47).

The next step is to obtain the involutive Hamiltonian. The generating functional $G_{\alpha}^{(n)}$ which determines this Hamiltonian is obtained from (28),

$$
\begin{gathered}
G_{\alpha}^{(0)}=\left\{\Theta_{\alpha}, H_{c}\right\} \\
G_{\alpha}^{(n)}=\left\{\tau_{\alpha}^{(1)}, \tilde{H}^{(n-1)}\right\}+\left\{\Theta_{\alpha}, \tilde{H}^{(n)}\right\}, \quad(n \geq 1)
\end{gathered}
$$

where $\tilde{H}^{(n)}$ is given in (29) and the original Hamiltonian $\tilde{H}^{(0)}$ is taken to be the canonical Hamiltonian (44). It is noteworthy that the general expression (28) reduces to the remarkably simple form (52) since there are only two terms $\Theta_{\alpha}$ and $\tau_{\alpha}^{(1)}$ in the expansion (10), which is the result of the judicious choice (47). Explicit calculations yield,

$$
\begin{gathered}
G_{1}^{(0)}=\Theta_{2} \\
G_{2}^{(0)}=m^{2} \partial_{i} A^{i} .
\end{gathered}
$$


which is substituted in (29) to obtain $\tilde{H}^{(1)}$,

$$
\tilde{H}^{(1)}=\int\left[\left(\partial_{i} A^{i}\right) \Phi^{1}-\frac{1}{2}\left(\partial_{i} \pi^{i}+m^{2} A_{0}\right) \Phi^{2}\right] d^{3} x
$$

This is inserted back in (52) to deduce $G_{\alpha}^{(1)}$ which then yields $\tilde{H}^{(2)}$ from (29),

$$
\tilde{H}^{(2)}=\int d^{3} x\left[-\frac{m^{2}}{8}\left(\Phi^{2}\right)^{2}-\frac{1}{2 m^{2}}\left(\partial_{i} \Phi^{1}\right)\left(\partial^{i} \Phi^{1}\right)\right]
$$

after which the series terminates. Thus the complete expression for the desired Hamiltonian is,

$$
\tilde{H}=H_{c}+\tilde{H}^{(1)}+\tilde{H}^{(2)}
$$

which, by construction, is strongly involutive,

$$
\left\{\tilde{H}, \tau_{\alpha}\right\}=0
$$

This completes the operatorial ('abelian') conversion of the original second class system (with Hamiltonian $H_{c}$ and constraints $\Theta_{\alpha}$ ) into first class (with Hamiltonian $\tilde{H}$ and constraints $\tau_{\alpha}$ ).

It is well known, however, that there exists the Stückelberg [9] mechanism whereby the second class Lagrangian (40) can be embedded into the corresponding first class theory by extending the configuration space,

$$
\mathcal{L}^{\prime}=-\frac{1}{4} F_{\mu \nu}^{2}+\frac{1}{2} m^{2}\left(A_{\mu}+\partial_{\mu} \theta\right)\left(A^{\mu}+\partial^{\mu} \theta\right)
$$

where $\theta$, the new field, is the Stückelberg scalar. The Lagrangian (59) is invariant under the gauge transformations,

$$
\begin{aligned}
A_{\mu} & \rightarrow A_{\mu}-\partial_{\mu} \alpha \\
\theta & \rightarrow \theta+\alpha
\end{aligned}
$$

and characterises a first class theory.

We now unravel the correspondence of the Hamiltonian approach with Stückelberg's formalism. The first step is to identify the new variables $\Phi^{1}$, $\Phi^{2}$ (in the Hamiltonian formalism) as a canonically conjugate pair $\left(\rho, \pi_{\rho}\right)$,

$$
\begin{aligned}
& \Phi^{1} \rightarrow m^{2} \rho \\
& \Phi^{2} \rightarrow \frac{2}{m^{2}} \pi_{\rho}
\end{aligned}
$$


as may be easily checked from (14) and (47). The phase space partition function is then given by the Faddeev formula [4],

$$
Z=\int \mathcal{D}\left(A_{\mu} \pi^{\mu} \rho \pi_{\rho}\right) \prod_{\alpha, \beta} \delta\left(\tau_{\alpha}\right) \delta\left(\Gamma_{\beta}\right) \operatorname{det}\left\{\tau_{\alpha}, \Gamma_{\beta}\right\} e^{i S}
$$

where,

$$
S=\int\left(\pi_{\mu} \dot{A}^{\mu}+\pi_{\rho} \dot{\rho}-\tilde{H}\right)
$$

with $\tilde{H}$, the involutive Hamiltonian (57), now expressed in terms of $\left(\rho, \pi_{\rho}\right)$ instead of $\Phi^{1}, \Phi^{2}$. The gauge conditions $\Gamma_{\beta}$ are chosen so that the determinant occurring in the functional measure is nonvanishing. Moreover $\Gamma_{\beta}$ are assumed to be independent of the momenta so that these may be considered as Faddeev-Popov type gauge conditions. We now perform the momentum integrations to pass on to the configuration space partition function. The $\pi_{0}$ integral is trivially performed by exploiting the delta function $\delta\left(\tau_{1}\right)=\delta\left(\pi_{0}+m^{2} \rho\right)$ in (62). The other delta function $\delta\left(\tau_{2}\right)$ is expressed by its Fourier transform (with Fourier variable $\xi$ ) and the Gaussian integral over $\pi_{\rho}$ performed. This yields the action,

$$
\begin{aligned}
S & =\int\left[\pi_{i} \dot{A}^{i}-\xi \partial_{i} \pi^{i}+\frac{m^{2}}{2}\left(\dot{\rho}^{2}+\xi^{2}-2 \dot{\rho} \xi\right)-\frac{1}{2} \pi_{i}^{2}+\frac{1}{4} F_{i j}^{2}\right. \\
& \left.+\frac{1}{2} m^{2} A_{i}^{2}+m^{2} \partial_{i} A^{i} \rho-\frac{m^{2}}{2} \partial_{i} \rho \partial^{i} \rho\right]
\end{aligned}
$$

Note that the $A_{0}$ term cancelled out. The integral over $A_{0}$, therefore, reduces to a trivial identity since one of the gauge conditions $\Gamma_{\beta}$ must involve $A_{0}$ to have a nonvanishing P.B. with the constraint $\tau_{1}$. The Gaussian integral over $\pi_{i}$ is finally performed and the Fourier variable $\xi$ relabelled as $\left(-A_{0}\right)$ to express the action in a covariant form,

$$
\begin{aligned}
S & =\int\left[-\frac{1}{4} F_{\mu \nu}^{2}+\frac{1}{2} m^{2} A_{\mu}^{2}-m^{2} \partial_{\mu} A^{\mu} \rho+\frac{m^{2}}{2} \partial_{\mu} \rho \partial^{\mu} \rho\right] \\
& =\int\left[-\frac{1}{4} F_{\mu \nu}^{2}+\frac{1}{2} m^{2}\left(A_{\mu}+\partial_{\mu} \rho\right)^{2}-m^{2} \partial_{\mu}\left(A^{\mu} \rho\right)\right]
\end{aligned}
$$

Ignoring the last (boundary) term we find that the Lagrangian corresponding to (65) agrees with the usual Stückelberg form (59) by identifying $\rho$ with the Stückelberg field $\theta$. There are, however, some interesting points concerning 
this correspondence between the Hamiltonian and Lagrangian embeddings which are now elaborated.

The first observation is that Stückelberg Lagrangian (59) (or, equivalently, considering (65) without the boundary term) leads to the primary constraint,

$$
T_{1}^{\prime}=\pi_{0} \approx 0
$$

The canonical Hamiltonian obtained by a Legendre transform is,

$$
H_{c}^{\prime}=\int\left[\frac{1}{2} \pi_{i}^{2}+\frac{\pi_{\rho}^{2}}{2 m^{2}}+\frac{1}{4} F_{i j}^{2}+\frac{m^{2}}{2}\left(A_{i}+\partial_{i} \rho\right)^{2}-A_{0} T_{2}^{\prime}\right]
$$

where $T_{2}^{\prime}$ is the secondary constraint,

$$
T_{2}^{\prime}=\partial_{i} \pi^{i}+\pi_{\rho} \approx 0
$$

No further constraints are present. It simple to see that $T_{1}^{\prime}$ and $T_{2}^{\prime}$ form a pair of first class constraints which are in (weak) involution with the total Hamiltonian. The system is first class which is precisley what one expects. The point to be emphasised is, however, that the set of constraints $\left(T_{1}^{\prime}, T_{2}^{\prime}\right)$ and the Hamiltonian (67) do not agree with the corresponding structures (49, 57, 61) obtained in the Hamiltonian approach. In the latter it is necessary to modify the structure of all the original second class constraints in (49) to make them first class. Here, however, only the 'Gauss' constraint is modified (68) while the other (66) is not. Indeed it can be checked that in the approach of BT [8] a partial modification of (cf. (66), (68)) would lead to an algebraic inconsistency. More specifically, the matrix $X_{\alpha \beta}$ (17) cannot be constructed. Consequently it is found that although it is possible to obtain the Stückelberg form (59) starting from the BT construction, the converse is not true.

The next question, naturally, is to find the Lagrangian compatible with the BT construction. Indeed we show that it is just the first expression in (65),

$$
\mathcal{L}^{\prime \prime}=-\frac{1}{4} F_{\mu \nu}^{2}+\frac{1}{2} m^{2} A_{\mu}^{2}-m^{2} \partial_{\mu} A^{\mu} \rho+\frac{m^{2}}{2}\left(\partial_{\mu} \rho\right)^{2}
$$

The primary constraint is,

$$
T_{1}^{\prime \prime}=\pi_{0}+m^{2} \rho \approx 0
$$


The canonical Hamiltonian obtained from (69) is

$$
\begin{aligned}
H & =+\frac{1}{2} \pi_{i}^{2}+\frac{\pi_{\rho}^{2}}{2 m^{2}}+\frac{1}{4} F_{i j}^{2}-\frac{1}{2} m^{2} A_{\mu}^{2} \\
& +m^{2} \partial_{i} A^{i} \rho-\frac{m^{2}}{2} \partial_{i} \rho \partial^{i} \rho-A_{0} \partial_{i} \pi^{i}
\end{aligned}
$$

Time conserving $T_{1}^{\prime \prime}(70)$ leads to the secondary constraint,

$$
T_{2}^{\prime \prime}=\partial_{i} \pi^{i}+m^{2} A_{0}+\pi_{\rho} \approx 0
$$

It can be checked that no further constraints are generated by this iterative scheme. Furthermore $T_{1}^{\prime \prime}, T_{2}^{\prime \prime}$ are a pair of first class constraints in involution with the Hamiltonian (71). It is important to note further that $T_{1}^{\prime \prime}, T_{2}^{\prime \prime}$ are exactly identical to the set of first class constraints (49, 61) obtained in the Hamiltonian formalism. Moreover the first class Hamiltonian (71) differs from the involutive Hamiltonian (57) by a term proportional to the first class constraint $\tau_{2}(49)$,

$$
H=\tilde{H}+\frac{\pi_{\rho}}{m^{2}} \tau_{2}
$$

Acting on physical states, this difference is trivial since such states are annihilated by the first class constraints. Similarly the equations of motion for observable (i.e. gauge invariant variables) will also be unaffected by this difference since $\tau_{2}$ can be regarded as the generator of the gauge transformations. In the construction of the functional integral this difference is inconsequential since the constraint $\tau_{2}$ is strongly implemented by the delta function $\delta\left(\tau_{2}\right)$ (62). Thus $H$ and $\tilde{H}$ may be regarded as canonically equivalent. This completes our demonstration of the compatibility of the Lagrangian (69) with the Hamiltonian description of BT.

To summarise, we emphasise the role played by the apparantly innocuous boundary term in (65). If we drop it, the resulting Lagrangian is exactly identical to the Stückelberg form (59) with the BT field identified with the Stückelberg scalar. In that case, however, the Hamiltonian and the set of constraints obtained from this Lagrangian are completely inequivalent to the original BT construction. If, on the contrary, the boundary term is retained it yields the embedded Lagrangian (69). The constraints and Hamiltonian following from this Lagrangian are completely equivalent to the original Hamiltonian embedding. In this case, therefore, the cycle - Hamiltonian to the Lagrangian to the Hamiltonian - closes. 


\section{A nonabelian example - The nonabelian Proca model}

In this section we consider the implication of the generalised canonical approach for a nonabelian model whose dynamics is governed by the Lagrangian density,

$$
\mathcal{L}=-\frac{1}{4} F_{\mu \nu}^{a} F^{\mu \nu, a}+\frac{1}{2} m^{2} A_{\mu}^{a} A^{\mu, a}
$$

which is just the nonabelian extension of the usual Proca model (40). The corresponding gauge group may be arbitrarily taken whose structure constants will be denoted by $f^{a b c}$. There is a primary constraint,

$$
T_{1}^{a}=\pi_{0}^{a} \approx 0
$$

which, conserved with the total Hamiltonian,

$$
H_{T}=H_{c}+\int u^{a}(x) \pi_{0}^{a}(x)
$$

where $H_{c}$ is the canonical Hamiltonian,

$$
H_{c}=\int\left[\frac{1}{2}\left(\pi_{i}^{a}\right)^{2}+\frac{1}{2} m^{2}\left(A_{i}^{a}\right)^{2}+\frac{1}{4}\left(F_{i j}^{a}\right)^{2}+\frac{m^{2}}{2}\left(A_{0}^{a}\right)^{2}-A_{0}^{a} T_{2}^{a}\right]
$$

yields a secondary constraint,

$$
T_{2}^{a}=\partial_{i} \pi^{i a}-g f^{a b c} \pi^{i b} A_{i}^{c}+m^{2} A_{0}^{a}
$$

The Poisson algebra of constraints,

$$
\begin{gathered}
\left\{T_{1}^{a}(x), T_{1}^{b}(y)\right\}=0 \\
\left\{T_{1}^{a}(x), T_{2}^{b}(y)\right\}=-m^{2} \delta^{a b} \delta(x-y) \\
\left\{T_{2}^{a}(x), T_{2}^{b}(y)\right\}=g f^{a b c} T_{2}^{c} \delta(x-y)-g m^{2} f^{a b c} A_{0}^{c} \delta(x-y)
\end{gathered}
$$

clearly illustrates that $T_{1}^{a}, T_{2}^{a}$ are a set of second class constraints. Note, particularly the equation (81). This algebra has been given incorrectly by Senjanovic [15] where the second term in the right hand side of (81) is missing. Cosequently the corresponding Dirac Brackets (involving $A_{0}^{a}$ ) have been 
incorrectly evaluated. The correct Dirac brackets may be computed from $(79-81)$ and are found to be,

$$
\begin{gathered}
\left\{\pi^{i a}(x), A_{0}^{b}(y)\right\}_{D B}=-\frac{g}{m^{2}} f^{a b c} \pi^{i c}(x) \delta(x-y) \\
\left\{\pi^{i a}(x), A_{j}^{b}(y)\right\}_{D B}=-\delta^{a b} \delta_{j}^{i} \delta(x-y) \\
\left\{\pi^{i a}(x), \pi_{j}^{b}(y)\right\}_{D B}=0 \\
\left\{A^{i a}(x), A_{j}^{b}(y)\right\}_{D B}=0 \\
\left\{A_{0}^{a}(x), A_{j}^{b}(y)\right\}_{D B}=\frac{1}{m^{2}} \partial_{j}^{x} \delta^{a b} \delta(x-y)-\frac{g}{m^{2}} f^{a b c} A_{j}^{c}(x) \delta(x-y) \\
\left\{A_{0}^{a}(x), A_{0}^{b}(y)\right\}_{D B}=-\frac{g}{m^{2}} f^{a b c} A_{0}^{c}(x) \delta(x-y) .
\end{gathered}
$$

There is one important difference in the Dirac brackets of the nonabelian theory (74) contrasted with the corresponding abelian version (40). In the former case these brackets are field dependent. Consequently a transition to quantum theory where these DBs are replaced by commutators will be problematic [1]. this was also mentioned earlier in our introduction. In this case, therefore, the generalised canonical formalism provides a viable alternative to quantisation. By converting the theory into first class, the need for Dirac brackets is eliminated and quantisation can proceed by using the canonical Poisson brackets.

As analysed in the previous section, the initial step is to obtain the first class constraints from the second class ones, (75) and (78). In order to construct the first class constraints $\tau_{\alpha}^{a}$ satisfying the involutive algebra (30) we have to specify the matrices $\omega_{a b}^{\alpha \beta}, X_{\alpha \beta}^{a b}$ which are the nonabelian analogues of (47) and (48)respectively. We make the following choice,

$$
\begin{gathered}
\omega_{a b}^{\alpha \beta}(x, y)=m^{2} \epsilon^{\alpha \beta} \delta^{a b} \delta(x-y) \\
X_{\alpha \beta}^{a b}(x, y)=\left(\begin{array}{lr}
2 \delta^{a b} & 0 \\
g f^{a b c} A_{0}^{c} & \frac{1}{2} \delta^{a b}
\end{array}\right) \delta(x-y)
\end{gathered}
$$

The corresponding inverse matrices are,

$$
\omega_{\alpha \beta}^{a b}(x, y)=\frac{1}{m^{2}} \epsilon_{\alpha \beta} \delta_{a b} \delta(x-y)
$$




$$
X_{a b}^{\alpha \beta}(x, y)=\left(\begin{array}{lr}
\frac{1}{2} \delta^{a b} & 0 \\
-g f^{a b c} A_{0}^{c} & 2 \delta^{a b}
\end{array}\right) \delta(x-y)
$$

We further take the following form for $\Gamma_{\alpha \beta \gamma}(x, y, z)$ appearing in (30),

$$
\Gamma_{\alpha \beta \gamma}(x, y, z)=g \delta_{\alpha 2} \delta_{\beta 2} \delta_{\gamma 2} \delta(x-y) \delta(y-z)
$$

so that (30) with (92) yields the conventional involutive algebra valid for the first class Yang-Mills theory.

With the choice (88), (89), (92) one can compute the generating functions $B_{\alpha \beta}^{a b}(32-35)$. From this knowledge the various terms (39) in the power series expansion (31) may be obtained. We give below the final expressions for the first class constraints $\tau_{1}^{a}, \tau_{2}^{a}$ satisfying (30), (92) valid upto the second power in the additional fields $\Phi^{\alpha, a}$ :

$$
\begin{gathered}
\tau_{1}^{a}=T_{1}^{a}+2 \Phi^{1, a} \\
\tau_{2}^{a}=T_{2}^{a}+\frac{1}{2} \Phi^{2, a}+g f^{a b c} \Phi^{1, b} A_{0}^{c}-\frac{g}{2 m^{2}} f^{a b c} \Phi^{2, b} \Phi^{1, c} \\
+\frac{g^{2}}{3 m^{2}} f^{a d b} f^{b c e} A_{0}^{e} \Phi^{1, c} \Phi^{1, d}+O(\Phi \Phi \Phi)
\end{gathered}
$$

where $T_{1}^{a}, T_{2}^{a}$ are the original second class constraints. Note further that $\tau_{1}^{a}$ is an exact result while $\tau_{2}^{a}$ gets modified in the different powers of the new fields $\Phi$.

The next step is to compute the involutive Hamiltonian. To complete the analogy with the usual Yang-Mills system we demand the following algebra to be satisfied by the involutive Hamiltonian $\tilde{H}$ and the constraints,

$$
\begin{aligned}
& \left\{\tau_{1}^{a}(x), \tilde{H}\right\}=\tau_{2}^{a}(x) \\
& \left\{\tau_{2}^{a}(x), \tilde{H}\right\}=g f^{a b c} A_{0}^{b} \tau_{2}^{c}
\end{aligned}
$$

As usual $\tilde{H}$ is expressed as a power series (23), with the original Hamiltonian $H_{0}$ taken to be the canonical piece. For algebraic considerations we mention that the old (second class) constraints and the original (canonical) Hamiltonian have identical brackets as (95), i.e.

$$
\begin{aligned}
& \left\{T_{1}^{a}(x), H_{c}\right\}=T_{2}^{a}(x) \\
& \left\{T_{2}^{a}(x), H_{c}\right\}=g f^{a b c} A_{0}^{b}(x) T_{2}^{c}(x)
\end{aligned}
$$


We now wish to compute the generating function $G_{\alpha}^{(n)}$ (28) which will yield the desired involutive Hamiltonian. Recall that the structure given in (28) leads to a strongly involutive Hamiltonian satisfying (21). Since our algebra (95) is only weakly involutive, suitable modifications must be made in the analysis given in section 2. Let us first express (95) in a covariant notation,

$$
\left\{\tau_{\alpha}^{a}(x), \tilde{H}\right\}=\int d y V_{\alpha \beta}^{a b}(x, y) \tau_{\beta}^{b}(y)
$$

so that,

$$
\begin{aligned}
& V_{12}^{a b}(x, y)=\delta^{a b} \delta(x-y) \\
& V_{22}^{a b}(x, y)=-g f^{a b c} A_{0}^{c} \delta(x-y)
\end{aligned}
$$

with all other $V$-coefficients being zero.

Proceeding as was done in sec 2, the modified expressions for the generating functions (28) are found to be,

$$
\begin{aligned}
& G_{\alpha}^{a(0)} \equiv\left\{\tau_{\alpha}^{a(0)}, \tilde{H}^{(0)}\right\}-\int d y V_{\alpha \beta}^{a b}(x, y) \tau_{\beta}^{b(0)}(y) \\
& G_{\alpha}^{a(1)} \equiv\left\{\tau_{\alpha}^{a(1)}, \tilde{H}^{(0)}\right\} \quad+\quad\left\{\tau_{\alpha}^{a(0)}, \tilde{H}^{(1)}\right\}+\left\{\tau_{\alpha}^{a(2)}, \tilde{H}^{(1)}\right\}_{(\Phi)} \\
& -\int d y V_{\alpha \beta}^{a b}(x, y) \tau_{\beta}^{b(1)}(y) \\
& G_{\alpha}^{a(n)} \equiv \sum_{m=0}^{n}\left\{\tau_{\alpha}^{a(n-m)}, \tilde{H}^{(m)}\right\}+\sum_{m=0}^{n-2}\left\{\tau_{\alpha}^{a(n-m)}, \tilde{H}^{(m+2)}\right\}_{(\Phi)}+\left\{\tau_{\alpha}^{a(n+1)}, \tilde{H}^{(1)}\right\}_{(\Phi)} \\
& -\int d y V_{\alpha \beta}^{a b}(x, y) \tau_{\beta}^{b(n)}(y), \quad n \geq 2
\end{aligned}
$$

The final solution for $\tilde{H}$ satisfying (97) is given by the power series (23) with,

$$
\tilde{H}^{(n+1)}=-\frac{1}{n+1} \int \Phi^{\mu, a} \omega_{\mu \nu}^{a b} X_{b c}^{\nu \rho} G_{\rho}^{c(n)}, \quad(n \geq 0)
$$

where $\omega_{\mu \nu}^{a b}, X_{b c}^{\nu \rho}$ are defined in (90), (91). The result for the involutive Hamiltonian now follows by a straightforward algebra,

$$
\tilde{H}=H_{c}-\frac{1}{m^{2}} \int \Phi^{1 a} \Phi^{2 a}-\frac{2 g}{m^{2}} f^{a b c} f^{c g e} \int A_{0}^{b} A_{0}^{g} \Phi^{1 a} \Phi^{1 e}+O(\Phi \Phi \Phi)
$$


correct to second powers in the new fields.

It is now possible to establish a connection of our analysis with the Lagrangian formalism [16] of converting (74) into first class. In [16] the gauge group has been chosen as $S U(2)$ for algebraic simplification. The configuration space is extended by introducing three additional fields which are just the Eulerian angles [16. This implies the addition of six new fields in the phase space (the fields of the configuration space and their canonically conjugate momenta). The new fields that we have introduced are precisely six in number for the group $S U(2)$. Three are given by $\Phi^{1, a}$ and three by $\Phi^{2, a}(a=1,2,3)$. The involutive algebra in the Lagrangian formulation [16] is exactly identical to (30) with $\Gamma_{\alpha \beta \gamma}$ specified by (92), and (95). Conceptually, therefore, the first class system found here by the Hamiltonian formalism corresponds to the Lagrangian scheme [16]. To make an exact correspondence which will equate some combination of the $\Phi^{1, a}, \Phi^{2, a}$ fields with the Eulerian fields (and their conjugates) is more of a technical problem. We will have to isolate the terms in the power series (94), (102) which will yield the corresponding terms in the trigonometric (sine, tan etc) expansions given in [16].

We will close this section by pointing out certain erroneous observations made in the literature [17] regarding the conversion of (74) to a first class system by adopting the usual Stückelberg 90 mechanism. Just as the abelian (Proca) model (40) could be converted into first class by making a 'gauge transformation' (see ),

$$
A_{\mu} \rightarrow A_{\mu}+\partial_{\mu} \theta
$$

it has been claimed [17] that a 'nonabelian gauge transformation'

$$
A_{\mu}^{a} \rightarrow A_{\mu}^{a}+\left(D_{\mu} \theta\right)^{a}
$$

is able to transform (74) into a first class system. This is wrong as we promptly demonstrate.

The Lagrangian (74) modified by (104) is,

$$
\mathcal{L}=-\frac{1}{4}\left(F_{\mu \nu}^{a}\right)^{2}+\frac{m^{2}}{2}\left(A_{\mu}^{a}+\left(D_{\mu} \theta\right)^{a}\right)^{2}
$$

There is one primary constraint,

$$
T_{1}^{a}=\pi_{0}^{a} \approx 0
$$


and one secondary constraint,

$$
T_{2}^{a}=\partial_{i} \pi_{i}^{a}+g f^{a b c} \pi_{i}^{b} A_{i}^{c}-m \pi_{\phi}^{a}-g f^{a b c} \pi_{\phi}^{b} \phi^{c}
$$

obtained by time conserving (106) with the total Hamiltonian,

$$
H_{T}=H_{c}+\int u^{a} \pi_{0}^{a}
$$

where $H_{c}$ is the canonical Hamiltonian,

$$
\begin{aligned}
H_{c} & =\frac{1}{2}\left(\pi_{i}^{a}\right)^{2}+\frac{1}{2}\left(A_{i}^{a}\right)^{2}+\frac{1}{4}\left(F_{i j}^{a}\right)^{2}+\frac{1}{2}\left(\partial_{i} \phi^{a}\right)^{2} \frac{1}{2}\left(\pi_{\phi}^{a}\right)^{2}+m A_{i}^{a} \partial_{i} \phi^{a} \\
& -m f^{a b c} A_{i}^{b} \phi^{c} \partial_{i} \phi^{a}+\frac{1}{2} g^{2} f^{a b c} f^{a d e} A_{i}^{b} A_{i}^{d} \phi^{c} \phi^{e}+A_{0}^{a} T_{2}^{a}
\end{aligned}
$$

The algebra of constraints,

$$
\begin{gathered}
\left\{T_{1}^{a}(x), T_{1}^{b}(y)\right\}=\left\{T_{1}^{a}(x), T_{2}^{b}(y)\right\}=0 \\
\left\{T_{2}^{a}(x), T_{2}^{b}(y)\right\}=g f^{a b c} T_{2}^{c} \delta(x-y)-g m^{2} f^{a b c} \pi_{\theta}^{c} \delta(x-y)
\end{gathered}
$$

clearly shows that $T_{2}^{a}$ is a second class constraint. Hence the model (105) is not first class. In fact, it is an example of a mixed class system because $T_{1}^{a}$ is first class. The relation in (111) was not computed in [17 which led its authors to incorrectly observe that (105) is a first class theory.

\section{Conclusion}

In the preceeding sections we have made a detailed investigation of the Batalin- Tyutin [8] method of converting second class systems into first class. Since the method has been developed quite recently it may not be familiar even among particle physicists. We have, therefore, reviewed this analysis and also shown the possibility of extending it in other directions. In particular, the method can be generalised to yield weakly involutive systems originating from second class systems. These weakly involutive systems occur naturally in nonabelian gauge theories and in gravity. The original paper [8] is more suited for discussing abelian models. 
The systematic application of this approach to specific models- both abelian and nonabelian- has revealed several interesting features and helps in giving new insights than would be possible by merely discussing the general formalism. The Proca model, which is an example of an abelian second class system, is systematically converted into first class. Interestingly, we find that the choice of the matrices $X_{i j}(47), \omega^{i j}(48)$, which was earlier used by us [19, 20] in other contexts, considerably simplifies the algebra. A direct connection with the usual Lagrangian embedding of Stückelberg [9] can be made by explicitly evaluating the momentum integrals in the phase space partition function using Faddeev-Popov [14]-like gauges. An exact identification of the extra field introduced in our Hamiltonian formalism with the conventional Stückelberg scalar [9] is possible provided one is careful with boundary terms (65). In this connection our analysis may be compared with [12], which is not only unsystematic (so may not conform to the strict mathematical rigour of quantising first class systems [3, 4, 5]) but whose correspondence with the Stückelberg mechanism is rather circuitous. We find that the present approach is more elegant and conceptually clean.

Coming next to the nonabelian extension of the Proca model we have first corrected['] the familiar Dirac analysis given in [15]. Using the generalised canonical approach, this model has been coverted into a first class system. Contrary to the abelian example, closed expressions for the Gauss constraint and the involutive Hamiltonian cannot be found. Conceptually it is possible to interpret the extra fields as the analogue of the Euler angles (regarded as field variables) introduced in the nonabelian $(S U(2))$ Stückelberg formalism [16]. It is also possible to obtain first class structure (of constraints, Hamiltonian etc) within the Hamiltonian formulation which are different from the conventional nonabelian Stückelberg mechanism [16]. Our analysis clearly reveals that, contrary to existing claim [17], the ususal (abelian) Stückelberg formalism [9] cannot transform the nonabelian Proca model into first class.

We feel that our nonabelian exercise may provide fresh insights in the fields of quantum gravity. The point is that it is possible, by suitably altering the generating fuctions, to express the constraint algebra as rank zero, rank one or rank two. It may be recalled that a strongly involutive algebra has

\footnotetext{
${ }^{2}$ Equation (81) given in [15] is wrong leading to an incorrect evaluation of the Dirac brackets involving $A_{0}^{a}$. For the correct forms, see (87)

${ }^{3}$ The algebra for $G^{a}$ immediately exposes the second class nature since it does not close, $\left\{G^{a}, G^{b}\right\}=\epsilon^{a b c} G^{c}+\cdots$.
} 
rank zero, an algebra which closes with structure constants has rank one; and an algebra which closes with structure functions of the phase space variables has rank two. In the case of quantum gravity Ashtekar [21] and Witten [22] have given algebras which fall in these clases. It would be tempting to discuss these aspects within the generalised canonical framework. 


\section{References}

[1] P. A. M. Dirac, "Lectures on quantum mechanics" (Belfer Graduate School, Yeshiva University Press, New York 1964).

[2] L. D. Faddeev, Theor. Math. Phys. 1 (1970), 1.

[3] E. S. Fradkin and G. A. Vilkovisky, Phys. Lett. B55 (1975) 224; CERN Report TH-2332 (1977) (unpublished).

[4] M. Henneaux, Phys. Rep. C126 (1985), 1, and "Classical foundations of BRST symmetry" (Bibliopolis, Naples, 1988).

[5] C. Becchi, A. Rouet and R. Stora, Ann. Phys. [N.Y.]98 (1976), 287

I. V. Tyutin, Lebedev Preprint 39 (1975).

[6] K. Kuchar, Phys. Rev. D34 (1986), 3044.

[7] I. A. Batalin and E. S. Fradkin, Nucl. Phys. B279 (1987), 514; Phys. Lett. B180 (1986), 157.

[8] I. A. Batalin and I. V. Tyutin, Int. J. Mod. Phys. A6 (1991), 3255.

[9] E. C. G. Stückelberg, Helv. Phys. Act. 30 (1957), 209.

[10] L. D. Faddeev and shatashvili, Phys. Lett. B167 (1986), 225.

[11] J. Wess and B. Zumino, Phys. Lett. B37 (1971), 95.

[12] T. Fujiwara, Y. Igarashi and J. Kubo, Nucl. Phys. B341 (1990), 695.

[13] Y. Kim, S. Kim, W. Kim, Y. Park, K. Kim and Y. Kim, Phys. Rev. D46 (1992), 4574

R. Banerjee, H. J. Rothe and K. D. Rothe, Phys. Rev. D48 (1994) R5467, (in Press)

[14] L. D. Faddeev and V. N. Popov, Phys. Lett. B25 (1967), 29.

[15] P. Senjanovic, Ann. Phys. [N. Y.] 100 (1976), 227.

[16] T. Kunimasa and T. Goto, Prog. Theo. Phys. 37 (1967), 452. 
[17] C. Grosse-Knetter, Phys. Rev. D48 (1993), 2854.

[18] R. Banerjee, Phys. Rev. D 48 (1993), R5467.

[19] N. Banerjee, S. Ghosh and R. Banerjee, Nucl. Phys. B (1994), (in Press).

[20] N. Banerjee, S. Ghosh and R. Banerjee, Phys. Rev. D 49 (1994), 1996.

[21] A. Ashtekar, Phys. Rev. D36 (1987), 1587.

[22] E. Witten, Nucl. Phys. B311 (1988), 46. 